\title{
BMJ Open Domains and outcome measures for the assessment of limited cutaneous systemic sclerosis: a scoping review protocol
}

\author{
Alain Lescoat (D) , ,2,3,4 David Roofeh, ${ }^{2,3}$ Whitney Townsend, ${ }^{5}$ Michael Hughes, ${ }^{6}$ \\ Robert D Sandler, ${ }^{6}$ François Zimmermann, ${ }^{1}$ John D Pauling, ${ }^{7}$ Maya H Buch (D) , 8,9 \\ Dinesh Khanna (1) 2,3
}

To cite: Lescoat A, Roofeh D, Townsend W, et al. Domains and outcome measures for the assessment of limited cutaneous systemic sclerosis: a scoping review protocol. BMJ Open 2021;11:e044765. doi:10.1136/ bmjopen-2020-044765

- Prepublication history for this paper is available online To view these files, please visit the journal online (http://dx.doi. org/10.1136/bmjopen-2020044765).

Received 14 September 2020 Revised 21 January 2021 Accepted 28 January 2021
Check for updates

(C) Author(s) (or their employer(s)) 2021. Re-use permitted under CC BY-NC. No commercial re-use. See rights and permissions. Published by BMJ.

For numbered affiliations see end of article.

Correspondence to

Dr Alain Lescoat;

alain.lescoat@chu-rennes.fr

\section{ABSTRACT}

Introduction Limited cutaneous systemic sclerosis (ICSSc) is the most frequent subset of systemic sclerosis. Despite this, IcSSc is not the major focus of clinical studies. The lack of interventional studies in ICSSc is due, in part, to a paucity of relevant outcome measures to effectively evaluate this subset. A combined response index dedicated to IcSSc would facilitate development of well-designed trials and approval of new drugs. The objective of this scoping review is to perform a broad and comprehensive identification of the outcome measures (core set items) within relevant domains, which have been used so far to assess ICSSC.

Methods and analysis The planned scoping review will be based on the approach proposed by Arksey et al and further developed by Levac et al. Development and reporting will follow the Preferred Reporting Items for Systematic Reviews and Meta-Analyses-Extension for Scoping Reviews checklist and guidelines. The development of the search strategy was guided by the concepts of domains and outcomes based on the Outcome Measures in Rheumatology approach and by the different names and definitions of SSc, with a specific emphasis on their occurrence in clinical trial studies. Two databases will be searched: MEDLINE and Embase. Studies in English, published from the year 1988 onwards, will be included, since 1988 corresponds to the publication of LeRoy's first consensus definition of ICSSC. Data will be extracted and analysed using a standardised charting tool.

Ethics and dissemination № ethical approval is required for this study. The results will be submitted to an international peer-reviewed journal and scientific conferences, informing the discussion on which items should be included in a combined response index dedicated to IcSSc (the CRISTAL project: Combined Response Index for Scleroderma Trial Assessing IcSSc).

\section{INTRODUCTION}

\section{Rationale}

Systemic sclerosis (SSc) or scleroderma is a rare autoimmune disorder which includes a wide range of clinical manifestations. ${ }^{1}$ SSc is characterised by the association of three

\section{Strengths and limitations of this study}

- The proposed scoping review will allow for comprehensive identification of the outcome measures (core set items) and relevant domains, which have been incorporated in the assessment of limited cutaneous systemic sclerosis (SSc).

- The limitations of the scoping review include the limited number of databases, the language and search terms used.

- This scoping review may also under-represent observational studies that do not explicitly mention the words 'limited SSc' or 'limited cutaneous SSc' in their title or abstract.

main features: vasculopathy with Raynaud's phenomenon as the hallmark symptom, exuberant fibrosis of the skin and internal organs, and immune activation with the occurrence of specific autoantibodies and inflammatory features such as synovitis and tenosynovitis. ${ }^{2}$

SSc is further subclassified into limited cutaneous (lcSSc) and diffuse cutaneous (dcSSc) according to 1988 LeRoy and Medsger's classification, ${ }^{3}$ revised in $2001,{ }^{4}$ and based on the extent of skin involvement by underlying fibrosis. LcSSc constitutes $60 \%-70 \%$ of all SSc and is considered a milder subphenotype of SSc with Raynaud's phenomenon and GI (Gastro-intestinal) involvement as common features and pulmonary arterial hypertension also observed in this subgroup. However, accumulating data from European Scleroderma Trials and Research group and other cohorts show that these and wider visceral complications occur in a significant proportion of patients with lcSSc. ${ }^{5}$ This concept is further highlighted by the recent clinical trials targeting interstitial lung disease that recruited patients with lcSSc as well as dcSSc 
(SLS studies (Scleroderma Lung Study I and II $)^{67}$ and SENSCIS trial ${ }^{8}$ (Safety and Efficacy of Nintedanib in Systemic Sclerosis). Moreover, recent studies focusing on quality of life have demonstrated that patients with lcSSc experience a significant impairment in their daily quality of life and that patients' experiences of 1cSSc have been largely overlooked. ${ }^{910}$

Despite this high prevalence of lcSSc and typically earlier diagnosis of this specific subtype, there is poor validation of dedicated strategies for the management of patients with lcSSc. The lack of interventional studies in lcSSc is due, in part, to a paucity of relevant outcome measures to effectively evaluate this major subset. The range of clinical manifestations of SSc is wide, and clinical items are classified within domains, for example, outcomes related to vasculopathy such as digital ulcers or outcomes related to interstitial lung disease such as the decline of pulmonary function measured through the annual decline of forced vital capacity (FVC). ${ }^{11}{ }^{12}$ Drug development and trials have focused on dcSSc partly due to the availability of validated outcome measures, including a relevant combined response index, the ACR-CRISS index (American College of Rheumatology-Combined Response Index for diffuse Systemic Sclerosis), ${ }^{131} 14$ that captures the global improvement of dcSSc. A composite index score dedicated to lcSSc that combines different aspects of the disease would similarly facilitate development of well-designed trials and approval of new drugs to treat lcSSc. ${ }^{15}$ Recent observational studies have highlighted the prognostic and predictive values of new imaging techniques and clinical markers such as capillaroscopy or laser doppler for vasculopathy, but the relevance of including such evaluation tools in combined index approach is still to be determined. Identifying and defining relevant candidate outcome measures within key SSc-associated domains ${ }^{16}$ to be included in such a combined index is the necessary first step for the construction of a future index for lcSSc.

\section{Objective}

The objective of this scoping review is to perform a broad and comprehensive identification of the core set items (and/or outcome measures) within relevant domains, which have been used so far to assess lcSSc since the endorsement of its consensual definition in 1988.

\section{METHODS AND ANALYSIS}

We have chosen to conduct a scoping review to perform this literature search, based on the methodological framework proposed by Arksey and O'Malley ${ }^{17}$ and further developed by Levac et al. ${ }^{18}$ Scoping reviews are especially effective to identify key factors/characteristics related to a concept, in our case, domains related to the assessment of lcSSc, and, to examine how research is conducted on a certain topic or field, in our case, the outcome measures within the identified domains. ${ }^{19}$ This scoping review will comprehensively identify outcome measures in lcSSc to inform on how lcSSc has been evaluated to date and identify gaps in domains of clinical relevance. This is the first step of a project which aims to select the items that could be included in a combined response index for clinical trials assessing patients with lcSSc (the CRISTAL project : Combined Response Index for Scleroderma Trial Assessing lcSSc). ${ }^{15}$

\section{Conceptual framework and key concepts}

The concepts of domains and outcomes are based on the Outcome Measures in Rheumatology approach. ${ }^{20}$ This approach is made up of two important and sequential components: identification of what to measure (domain set), for example, in the field of SSc, measuring the impact of 'vasculopathy', measuring 'interstitial lung disease' or impact of pain on quality of life and then identification of how to measure each of the identified domains using relevant instruments or tools (outcome measurement set), that is, for the domain 'vasculopathy', the number of new digital ulcers occurring during follow-up or for the domain 'interstitial lung disease' change in FVC during the considered period or pain visual analogue scale or PROMIS (Patient-Reported Outcomes Measurement Information System) items to assess the intensity of pain and pain interference. ${ }^{21}$

The systematic identification of outcome measures (how to measure a manifestation/visceral involvement) and the domains they are related to (which manifestations of the disease/visceral involvement is measured) will inform on how lcSSc has been assessed to date and to guide the discussion on which items should be included in a combined response index dedicated to lcSSc.

\section{Publication dates and time period}

In 1988, LeRoy's classification of SSc, built on previous 1980 criteria, crystallised the two main subsets of SSc, lcSSc and dcSSc. Prior to LeRoy's classification, the concept of limited SSc was recognised, but several terms were used to describe features of this subgroup such as acrosclerosis, CREST (Calcinosis-Raynaud's phenomenon-Esophageal dysfunction-Sclerodactyly-Telangiectasia), dermatosclerosis or acroscleroderma. These definitions were inconsistent and in contrast with those of generalised scleroderma or diffuse scleroderma, with the latter more or less matching with the definition of the diffuse cutaneous subset. ${ }^{22}$ The absence of a consensus classification that fully captured the concept and components of limited SSc led to variable outcome measures and subgrouping criteria. The endorsement to define these two subsets (limited vs diffuse) of the disease within the 1988 classification criteria was based on prognostic data and defined by the extent of skin fibrosis involvement. This was a historical milestone in the nosology of SSc. Examining articles published before 1988 could lead to the inclusion of outcomes used to assess populations that would not match with the contemporary definition of lcSSc and have therefore not been included. After 1988, the term CREST/CRST (Calcinosis-Raynaud's phenomenon-Sclerodactyly-Telangiectasia) syndrome 
Table 1 Inclusion and exclusion criteria for the scoping review

\begin{tabular}{|c|c|}
\hline Inclusion criteria & Exclusion criteria \\
\hline $\begin{array}{l}\text { Language: English } \\
\text { Publication date: after } 1988 \text { (or in 1988) } \\
\text { Population: }\end{array}$ & Population \\
\hline $\begin{array}{l}\text { For observational studies: titles/abstract mentioning both } \\
\text { IcSSc and dcSSc will be kept, articles mentioning IcSSc } \\
\text { only, SSc sine scleroderma, limited SSc, CREST/CRST only } \\
\text { will be kept as well. When the number of IcSSc patients is } \\
\text { mentioned, only studies with } 20 \text { IcSSc patients or more will } \\
\text { be included. }\end{array}$ & $\begin{array}{l}\text { Articles only focusing on localised scleroderma/morphea } \\
\text { without including systemic sclerosis/systemic scleroderma } \\
\text { patients will be excluded, articles only focusing on VEDOSS } \\
\text { only, will be excluded as well. Articles focusing on dcSSc only } \\
\text { will be excluded. Studies not concerned with human subjects } \\
\text { or not pertaining to adult will be excluded. }\end{array}$ \\
\hline
\end{tabular}

For clinical/therapeutic trials: Titles/abstract that only mention systemic sclerosis/scleroderma without specifying dcSSc or IcSSc will be kept, and articles mentioning both IcSSc and dcSSc will be kept, articles mentioning lcSSc only, SSc sine scleroderma, CREST/CRST or limited SSc will be kept as well.

Studies

For observational studies: observational analytical crosssectional or longitudinal studies, case-control studies, prospective and retrospective cohort studies, randomised controlled trials, non-randomised controlled trials, Metaanalyses and systematic reviews.
For clinical/therapeutic trials: randomised controlled trials and non-randomised controlled trials will be considered, unrandomised or uncontrolled interventional or observational studies (before and after studies in routine care) or clinical trials evaluating a treatment or a therapeutic strategy will also be considered for full-text review. Meta-analysis and systematic reviews of clinical/therapeutic trials will also be included for full-text review.

\section{Studies}

For observational studies: narrative and non-systematic reviews, conference abstracts, biography, case report, comment, editorial, directory, festschrift, interviews, lectures, legal cases, legislation, letter, news, newspaper article, patient education handout, popular works, congresses, consensus development conference and practice guideline will be excluded. Genetic or epigenetic studies will be excluded. Observational studies specifically evaluating the prevalence of the disease will also be excluded. Translational and basic sciences studies, including observational studies dedicated to autoantibody testing, will not be considered for full-text review as they will not allow to identify new validated outcome measures reflecting feeling, functioning or surviving that could be used for an acceptable combined response index.

For clinical/therapeutic trials: Narrative reviews focusing on clinical trials, conference abstracts, biography, case report, comment, editorial, directory, festschrift, interviews, lectures, legal cases, legislation, letter, news, newspaper article, patient education handout, popular works, congresses, consensus development conference and practice guideline will be excluded.

For all: studies without abstract available (only title provided) will be excluded.

dcSSc, diffuse cutaneous systemic sclerosis; IcSSc, limited cutaneous systemic sclerosis; SSc, systemic sclerosis; VEDOSS, very early diagnosis of systemic sclerosis.

persisted and overlapped with lcSSc. Based on this, articles only mentioning CREST/CRST in their title and abstract after 1988 will also be considered for full-text review, and full-text assessment will confirm whether the population studies match with the contemporary definition of lcSSc.

\section{Scoping review questions}

Main question

What are the outcome measures within relevant domains that have been used to assess 1cSSc since the 1988 LeRoy's classification has been in use?

\section{Secondary questions}

How many studies have been published by year?

What types of studies have been published?

\section{General overview of the search strategy}

As this scoping review focuses on $1 \mathrm{cSSc} /$ scleroderma, our search terms will focus on studies with original data/original articles that explicitly mention the subtype 'limited' and/or CR(E)ST in their title or abstract (\#1). Nonetheless, when applying this strategy to milestone articles based on the reviewers' expertise ${ }^{6-8} 23-32$ we identified a gap, particularly in picking up clinical trials. Indeed, many clinical trials only mention 'scleroderma' in their title or abstract, without specifying limited or diffuse, although they indeed include patients with lcSSc. This is a major issue since the objective is to identify outcome measures to be included in a combined response index for clinical 
trials. To tackle this issue, we will include in the search terms all clinical trials mentioning scleroderma or SSc in the title or abstract (\#2), even if the word 'limited' is not mentioned in the title or abstract. For pragmatic reasons, observational studies will not be included in this \#2, only clinical trials, in line with the overall objective of this scoping review.

\section{Information sources}

Electronic databases: PubMed (MEDLINE), Embase. com.

\section{Search terms}

Final search strategy for title $/$ abstract evaluation $=\# 1$ and \#2 as follow ${ }^{33}$ :

\section{$\# 1$}

\section{Population}

\section{Search terms}

\section{Ovid MEDLINE sensitive}

exp Scleroderma, Limited/ OR (Scleroderma, Systemic/ AND limited.ti.) OR ((Systemic scleroderma.mp. OR systemic sclerodermas.mp. OR systemic sclerosis.mp. OR systemic scleroses.mp. OR SSc.mp.) ADJ3 limited.mp.) OR lcSSc.mp. OR ((Crest.ti,ab. OR CRST.ti,ab.) ADJ1 syndrome*.ti,ab.)

\section{Embase.com}

( 'limited scleroderma'/exp OR ('systemic sclerosis') de AND limited:ti) OR (('systemic scleroderma' OR 'systemic sclerodermas' OR 'systemic sclerosis' OR 'systemic scleroses' OR ssc) NEAR/3 limited):ti,ab) OR lcssc:ti,ab OR 'syndrome CREST'/exp OR (( (crest OR crst) NEAR/1 syndrome*):ti,ab)) NOT ([animals]/lim NOT [humans]/lim)) AND ('article'/it OR 'article in press'/it)

\section{Included}

- Titles/abstract mentioning both $1 \mathrm{cSS} c$ and dcSSc will be kept, articles mentioning lcSSc only, SSc sine scleroderma, limited SSc, CREST/CRST only will be kept as well. When the number of lcSSc patients is mentioned, only studies with $20 \mathrm{lcSSc}$ patients or more will be included.

\section{Excluded}

- Articles only focusing on localised scleroderma/ morphea without including systemic sclerosis/ systemic scleroderma patients will be excluded, articles that only mention systemic sclerosis/scleroderma without specifying dcSSc or lcSSc will be excluded, articles focusing on very early diagnosis of systemic sclerosis (VEDOSS) ${ }^{34}$ only and articles focusing on dcSSc only will be excluded as well.

Intervention: $\mathrm{n} / \mathrm{a}$.

Comparison: $\mathrm{n} / \mathrm{a}$.

Outcomes: $\mathrm{n} / \mathrm{a}$ as the selection of domains and outcome measures is the aim of this scoping review.
Studies

\section{Included articles}

- Studies written in English.

- Original studies including: observational analytical cross-sectional or longitudinal studies, case-control studies, prospective and retrospective cohort studies, randomised controlled trials, non-randomised controlled trials, before and after studies, metaanalyses and systematic reviews.

\section{Excluded articles}

- Narrative and non-systematic reviews, conference abstracts, biography, case report, comment, editorial, directory, festschrift, interviews, lectures, legal cases, legislation, letter, news, newspaper article, patient education handout, popular works, congresses, consensus development conference and practice guideline will be excluded. Genetic or epigenetic studies will be excluded. Observational studies specifically evaluating the prevalence of the disease will also be excluded. Translational and basic sciences studies, including observational studies dedicated to autoantibody testing, will not be considered for full-text review as they will not allow to identify new validated outcome measures reflecting feeling, functioning or surviving that could be used for an acceptable combined response index.

- Studies not concerned with human subjects or not pertaining to adult studies will be excluded.

- Article published before 1988 and LeRoy's classification (official creation/endorsement of the concept of limited SSc) will be excluded.

\#2

Population

Search terms

Ovid MEDLINE sensitive

using Sensitivity/precision maximised Cochrane limit*

(Exp Scleroderma, Systemic/OR "Systemic scleroderma".mp. OR "systemic sclerodermas".mp. OR "systemic sclerosis".mp. OR "systemic scleroses".mp.) AND

*Sensitivity/precision maximised Cochrane filter

(randomized controlled trial.pt. OR controlled clinical trial.pt. OR randomized.ab. OR placebo.ab. OR clinical trials as topic.sh. OR randomly.ab. OR trial.ti.) not (exp animals/ not humans.sh.)

\section{Included}

- Titles/abstract that only mention SSc/scleroderma without specifying dcSSc or lcSSc will be kept and articles mentioning both lcSSc and dcSSc will be kept; articles mentioning lcSSc only, SSc sine scleroderma, CREST/CRST or limited SSc will be kept as well.

\section{Excluded}

- Articles only focusing on localised scleroderma/ morphea without including systemic sclerosis/ systemic scleroderma patients will be excluded, 
and articles only focusing on VEDOSS only will be excluded as well. Articles focusing on dcSSc only will be excluded.

Intervention: randomised controlled trials and unrandomised controlled trials only.

Comparison: $\mathrm{n} / \mathrm{a}$.

Outcomes: $\mathrm{n} / \mathrm{a}$ as the selection of domains and outcome measures is the aim of this scoping review.

\section{Studies}

\section{Included articles}

- Only studies written in English will be considered.

- Randomised controlled trials and unrandomised controlled trials will be especially considered for this \#2. Unrandomised or uncontrolled interventional or observational studies (before and after studies in routine care) or clinical trials evaluating a treatment or a therapeutic strategy will also be considered for full-text review. Meta-analysis and systematic reviews of therapeutic trials will also be included for full-text review.

\section{Excluded articles}

- Reviews, conference abstracts, biography, case report, comment, editorial, directory, festschrift, interviews, lectures, legal cases, legislation, letter, news, newspaper article, patient education handout, popular works, congresses, consensus development conference and practice guideline will be excluded, observational analytical cross-sectional studies, case-control studies, prospective and retrospective cohort studies will be excluded.

- Studies not concerned with human subjects or not pertaining to adults will be excluded.

- Article published before 1988 and LeRoy's classification (official creation/endorsement of the concept of limited SSc) will be excluded.

\section{Synthesis of eligibility criteria}

\section{Inclusion criteria}

1. Language: English.

2. Publication date: after 1988 and Leroy's classification.

3. Population.

\section{For observational studies}

Titles/abstract mentioning both lcSSc and dcSSc will be kept, articles mentioning lcSSc only, SSc sine scleroderma, limited SSc and CREST/CRST only will be kept as well. When the number of lcSSc patients is mentioned, only studies with 20 lcSSc patients or more will be included.

\section{For clinical/therapeutic trials}

Titles/abstract that only mention SSc/scleroderma without specifying dcSSc or lcSSc will be kept, and articles mentioning both lcSSc and dcSSc will be kept, articles mentioning lcSSc only, SSc sine scleroderma, CREST/ CRST or limited SSc will be kept as well.

4. Studies
For observational studies observational analytical crosssectional or longitudinal studies, case-control studies, prospective and retrospective cohort studies, randomised controlled trials, non-randomised controlled trials, metaanalyses and systematic reviews.

\section{For clinical/therapeutic trials}

Randomised controlled trials and non-randomised controlled trials will be considered, unrandomised or uncontrolled interventional or observational studies (before and after studies in routine care) or clinical trials evaluating a treatment or a therapeutic strategy will also be considered for full-text review. Meta-analysis and systematic reviews of clinical/therapeutic trials will also be included for full-text review.

\section{Exclusion criteria}

\section{Population}

Articles only focusing on localised scleroderma/ morphea without including SSc/systemic scleroderma patients will be excluded, articles only focusing on VEDOSS only, will be excluded as well. Articles focusing on dcSSc only will be excluded. Studies not concerned with human subjects or not pertaining to adult will be excluded.

\section{Studies}

\section{For observational studies}

Narrative and non-systematic reviews, conference abstracts, biography, case report, comment, editorial, directory, festschrift, interviews, lectures, legal cases, legislation, letter, news, newspaper article, patient education handout, popular works, congresses, consensus development conference and practice guideline will be excluded. Genetic or epigenetic studies will be excluded. Observational studies specifically evaluating the prevalence of the disease will also be excluded. Translational and basic sciences studies, including observational studies dedicated to autoantibody testing, will not be considered for full-text review as they will not allow to identify new validated outcome measures reflecting feeling, functioning or surviving, that could be used for an acceptable combined response index.

\section{For clinical/therapeutic trials}

Narrative reviews focusing on clinical trials, conference abstracts, biography, case report, comment, editorial, directory, festschrift, interviews, lectures, legal cases, legislation, letter, news, newspaper article, patient education handout, popular works, congresses, consensus development conference and practice guideline will be excluded.

\section{For all}

Studies without abstract available (only title provided) will be excluded (table 1 ).

\section{Reporting of protocol and study records}

This study protocol follows PRISMA-ScR guidelines ${ }^{35}$ and PRISMA guidelines for the publication of systematic 
Table 2 General template for title and abstract screening

\section{Questions}

1. Is the article written in English?
2. Is the article after 1988 (or published in 1988)? $\square$ Yes $\square$ No
3. Is this an observational study based on primary $\quad \square$ Yes $\square$ No
data or is this a systematic review/metanalysis
published as original article, And is not a genetic
or epigenetic or prevalence or basic science/
biomarker study?

- If yes, if the number of patients from the above $\square$ Yes $\square$ No mentioned subgroup is specified, is it 20 or $\quad$ Yes $\square$ No more?

- If 1-2-3 fulfilled, does title or abstract mention $\square$ Uncertain: IcSSc or sine or ISSc or CREST/CRST? needs full-text reviewing

$\begin{array}{ll}\text { If 1-2 fulfilled and if the title or abstract does not } & \square \text { Yes } \square \text { No } \\ \text { mention IcSSc or sine or ISSc or CREST/CRST, } & \square \text { Uncertain: } \\ \text { Which does not exclusively concern dcSSc } & \text { needs full text } \\ \text { patients ? } & \text { reviewing } \\ \text { is this a randomizedrandomised controlled } & \\ \text { trial, non-randomizedrandomised controlled } & \\ \text { trial, an unrandomiszed or uncontrolled } & \\ \text { interventional or observational studies (before } & \\ \text { and after studies in routine care) or clinical } & \\ \text { trial evaluating a treatment or a therapeutic } & \\ \text { strategy? }\end{array}$

dcSSc, diffuse cutaneous systemic sclerosis; IcSSc, limited cutaneous systemic sclerosis; ISSc, limited systemic sclerosis.

review protocols,${ }^{36}$ with specific adaptations for this scoping review protocol.

Data management will be housed through Covidence (https://www.covidence.org/home), under the supervision of DK and MHB.

DR, JP, MH, RS, AL and FZ will screen citations and review for eligibility and inclusion based on the eligibility criteria and the article selection template (tables 1 and 2). AL will assess all the titles (T) and abstracts (A), and the other five reviewers will evaluate $1 / 5$ th of $\mathrm{T} / \mathrm{A}$ to ensure that all articles will be double checked. Inter-rater agreement will be evaluated using Cohen's Kappa statistics. A first test of agreement will be performed based on 50 citations. If Kappa coefficients are under 0.8, we will evaluate the disagreements and understand the reason to correct misunderstanding and ensure consistency for the rest of the review process. $\mathrm{T}$ and $\mathrm{A}$ will then be reviewed for the entire article list. Any disagreements between reviewers will be reviewed and resolved by DK and/or MHB. If uncertainty persists, the manuscript will be included for comprehensiveness. Where there is lack of data clarity pertaining to exclusion criteria in manuscripts, mentors will be contacted to discuss this issue. Agreement between pairs for overlapping citations will also be assessed using Cohen's Kappa statistics at the end of the process. After article selection, the data extraction template (table 3) for full-text review will be evaluated by two reviewers on a sample of $20 \%$ of included studies to adjust its sensitivity. Once the template is finalised, one reviewer will then perform the analysis, and the second reviewer will
Table 3 Preliminary charting table for data extraction

\begin{tabular}{ll}
\hline Item & Description \\
\hline Journal &
\end{tabular}

First author

Year publication

Patient population

$\square$ dcSSc and IcSSc (including ISSc, sine and CREST)

$\square$ IcSSc only (including ISSc, sine and CREST)

Number of patients evaluated

(total)

Number of patients with IcSSc

(including ISSc, sine and CREST)

$\begin{array}{ll}\text { Study type } \quad & \square \text { Observational cross-sectional } \\ \text { study (pro or retrospective) } \\ \square \text { Observational longitudinal study } \\ \text { (pro or retrospective) } \\ \square \text { Case-control study } \\ \square \text { Randomised clinical trial } \\ \square \text { Unrandomised clinical trial } \\ \square \text { Basic sciences (biomarkers) }\end{array}$

Domain 1 as explicitly mentioned in the article

Outcome 1 (of D1) with assessment methods

Outcome 2 (of D1) with assessment methods

Add as many outcomes as necessary

(...)

Domain 2 as explicitly

mentioned in the article

Outcome 1 (of D2) with

assessment methods

Outcome 2 (of D2) with

assessment methods

Add as many outcomes as

necessary

\section{(...)}

No domain explicitly

mentioned in the article (Dn/a)

Outcome 1 (of Dn/a) with

assessment methods

Outcome 2 (of Dn/a) with

assessment methods

Add as many outcomes as

necessary

(...)

dcSSc, diffuse cutaneous systemic sclerosis; IcSSc, limited cutaneous systemic sclerosis; ISSc, limited systemic sclerosis.

independently check a sample of the total of $20 \%$ articles, for accuracy. Any disagreements will be reviewed and resolved by DK and/or MHB. Citation searching will be applied to identify additional studies through checking 
of reference lists of primary studies. Although quality appraisal is typically necessary for systematic literature reviews and meta-analyses, using evaluation tools such as the Jadad scale, ${ }^{37}$ the Cochrane risk of bias tool ${ }^{38}$ or the Newcastle-Ottawa Scale for non-randomised studies ${ }^{39}$ our focus is to conduct a scoping review to determine domains and outcome measures that have been studied in lcSSc. As such, quality appraisal is not planned. We intend to start title and abstract selection on Covidence starting January 2021.

\section{Presentation of the results}

We expect to present the main results of this scoping review, with a least one table summarising domains and identified outcomes. For the main domains of interest, their frequency in the literature will also be provided in a table. Identified gaps and main issues concerning the lack of outcome measures and/or their poor use will also be highlighted in a third table. In the end, a comprehensive map of the main domains and outcomes will be provided within a dedicated graphical abstract or figure.

\section{ETHICS AND DISSEMINATION}

This scoping review is based on the analysis of published scientific literature without involving any patient, any new clinical or fundamental research or any type of personal information. Therefore, no ethical approval is required. The results of this scoping review will be submitted for publication in a peer-reviewed journal and will provide an overview of domains and items that are captured in observational cohorts and clinical trials in lcSSc and can be used for a combined index. The results concerning these domains and items, and each step of the creation of this combined index will also be submitted for presentation in international congresses of rheumatology.

\section{Patient and public involvement}

This scoping review plans to analyse the published scientific literature; no patients are involved for this specific analysis. The overall objective of the CRISTAL project is to develop a combined response index for lcSSc with input from the patient partners, clinicians with expertise in SSc and methodologists. All the steps of the project and patient partners involvement have been published previously. ${ }^{15}$

\section{STRENGTHS, LIMITATIONS AND DISCUSSION POINTS}

- The proposed scoping review will allow a broad and comprehensive identification of the outcome measures (core set items) within relevant domains, which have been used so far to assess lcSSc. The specific emphasis on clinical trials including patients with lcSSc will ensure the identification of relevant outcome measures used so far and their mapping within key domains, eventually highlighting gaps and main issues concerning the lack of outcome measures and/or their poor use.

- Although comprehensive, this study design has limitations regarding the number of databases, the language and search terms used and may underrepresent observational studies that do not explicitly mention the word 'limited SSc' or 'limited cutaneous SSc' in their title or abstract.

- By focusing on articles published after 1988, this scoping review may not capture the outcome measures used to assess equivalent subsets of lcSSc before this date, but this will ensure that the population included match with the contemporary definition of lcSSc.

- For this first phase of the initiative, we are seeking to identify relevant domains and outcomes as opposed to evaluate their specific properties in lcSSc. Nevertheless, the several clinical trials to be evaluated will not report results solely in lcSSc patients, which constitutes a limitation of our protocol.

- Regulatory agencies emphasise outcomes should reflect how patients feel, function and/or survive. With this in mind, as the main objective is to identify domains and outcomes that could be incorporated in a combined response index, we have not included congress databases or studies on exploratory biomarkers or epigenetic/genetic studies. Such a selection will limit the comprehensiveness of this scoping review but will insure its coherence with the global objective of the project. Regarding this main objective, to remain consistent with the comprehensive concept of scoping review, and considering that we are not performing a systematic review or metaanalysis, we will not evaluate quality appraisal, and this could be considered as a limitation of this protocol.

\section{Author affiliations}

${ }^{1}$ Internal Medicine \& Clinical Immunology, CHU Rennes, Rennes, France

${ }^{2}$ Scleroderma Program, University of Michigan, Ann Arbor, Michigan, USA

${ }^{3}$ Department of Internal Medicine, Division of Rheumatology, University of Michigan, Ann Arbor, Michigan, USA

${ }^{4}$ Univ Rennes, CHU Rennes, Inserm, EHESP, Irset (Institut de Recherche en Santé, Environnement et Travail) - UMR_S 1085, Rennes, France

${ }^{5}$ Taubman Health Sciences Library, University of Michigan, Ann Arbor, Michigan, USA ${ }^{6}$ Department of Rheumatology, Royal Hallamshire Hospital, Sheffield Teaching Hospitals NHS Foundation Trust, Sheffield, UK

${ }^{7}$ Department of Rheumatology, Royal National Hospital For Rheumatic Diseases (at Royal United Hospitals), University of Bath, Bath, UK

${ }^{8}$ Centre for Musculoskeletal Research, School of Biological Sciences, Faculty of Biology, Medicine \& Health, University of Manchester, Manchester, UK

${ }^{9}$ NIHR Manchester Biomedical Research Centre, Manchester Academic Health Science Centre, Manchester University Foundation Trust, Manchester, UK

Acknowledgements The authors thank Rachel Wessel from the scleroderma program of University of Ann Arbor (Michigan, USA) for her administrative support.

Contributors $A L$ and MHB wrote the first draft of the manuscript. AL, MHB, WT, DR, $\mathrm{JP}, \mathrm{MH}, \mathrm{RS}, \mathrm{FZ}$ and DK conceived the scoping review and developed the research questions and the search strategy. All authors critically reviewed drafts and edited the manuscript.

Funding This project has received funding from SRUK (Scleroderma and Raynaud UK) \& WSF (World Scleroderma Foundation) (UH\&UHR1) and additional support is 
provided by the National Institutes of Health/NIAMS grant to Dr DK (K24 AR063120). Dr DR was funded by the NIH/NIAMS T32 grant (AR007080). Dr AL was funded by the French network of the University Hospitals HUGO (Hôpitaux Universitaire du Grand Ouest) (AAP JCM2020) and by the 2020 CORECT grant from the Rennes University Hospital (France).

Competing interests $\mathrm{MH}$ has received speaker honoraria $(<\$ 10000)$ from Actelion pharmaceuticals, JP has received speaker's honoraria and research grant support (>\$10 000) from Actelion pharmaceuticals. JP has undertaken consultancy work for Actelion pharmaceuticals, Sojournix Pharma and Boehringer Ingelheim, MHB has received meeting support from Boehringer Ingelheim, AL, DR, WT, RS and FZ have no conflict of interest, DK is a consultant to Acceleron, Abbvie, Actelion, Amgen, Bayer, BMS, Boehringer Ingelheim, CSL Behring, Corbus, Galapagos, Genentech/Roche, GSK, Horizon, Mitsubishi Tanabe Pharma, Sanofi-Aventis and United Therapeutics. He has stock options in Eicos Sciences, Inc.

Patient and public involvement Patients and/or the public were not involved in the design, or conduct, or reporting or dissemination plans of this research.

Patient consent for publication Not required.

Provenance and peer review Not commissioned; externally peer reviewed.

Open access This is an open access article distributed in accordance with the Creative Commons Attribution Non Commercial (CC BY-NC 4.0) license, which permits others to distribute, remix, adapt, build upon this work non-commercially, and license their derivative works on different terms, provided the original work is properly cited, appropriate credit is given, any changes made indicated, and the use is non-commercial. See: http://creativecommons.org/licenses/by-nc/4.0/.

\section{ORCID iDs}

Alain Lescoat http://orcid.org/0000-0003-2081-8558

Maya H Buch http://orcid.org/0000-0002-8962-5642

Dinesh Khanna http://orcid.org/0000-0003-1412-4453

\section{REFERENCES}

1 Denton CP, Khanna D. Systemic sclerosis. Lancet 2017;390:1685-99.

2 Lescoat A, Ballerie A, Belhomme N, et al. Synovial involvement assessed by power Doppler ultra-sonography in systemic sclerosis: results of a cross-sectional study. Rheumatology 2018;57:2012-21.

3 LeRoy EC, Black C, Fleischmajer R, et al. Scleroderma (systemic sclerosis): classification, subsets and pathogenesis. J Rheumatol 1988;15:202-5.

4 LeRoy EC, Medsger TA. Criteria for the classification of early systemic sclerosis. J Rheumatol 2001;28:1573-6.

5 Frantz C, Huscher D, Avouac J, et al. Outcomes of limited cutaneous systemic sclerosis patients: results on more than 12,000 patients from the EUSTAR database. Autoimmun Rev 2020;19:102452.

6 Tashkin DP, Elashoff R, Clements PJ, et al. Cyclophosphamide versus placebo in scleroderma lung disease. $N$ Engl J Med 2006;354:2655-66.

7 Tashkin DP, Roth MD, Clements PJ, et al. Mycophenolate mofetil versus oral cyclophosphamide in scleroderma-related interstitial lung disease (SLS II): a randomised controlled, double-blind, parallel group trial. Lancet Respir Med 2016;4:708-19.

8 Distler O, Highland KB, Gahlemann M, et al. Nintedanib for systemic sclerosis-associated interstitial lung disease. $N$ Engl J Med 2019;380:2518-28.

9 Frantz C, Avouac J, Distler O, et al. Impaired quality of life in systemic sclerosis and patient perception of the disease: a large international survey. Semin Arthritis Rheum 2016;46:115-23.

10 Khanna D, Ahmed M, Furst DE, et al. Health values of patients with systemic sclerosis. Arthritis Rheum 2007;57:86-93.

11 Khanna D, Lovell DJ, Giannini E, et al. Development of a provisional core set of response measures for clinical trials of systemic sclerosis. Ann Rheum Dis 2008;67:703-9.

12 Boers M, Kirwan JR, Wells G, et al. Developing core outcome measurement sets for clinical trials: OMERACT filter 2.0. J Clin Epidemiol 2014;67:745-53.

13 Khanna D, Berrocal VJ, Giannini EH, et al. The American College of Rheumatology provisional composite response index for clinical trials in early diffuse cutaneous systemic sclerosis. Arthritis Rheumatol 2016;68:299-311.

14 Khanna D, Huang S, CJF L. New composite endpoint in early diffuse cutaneous systemic sclerosis: revisiting the provisional American College of rheumatology composite response index in systemic sclerosis. Ann Rheum Dis.
15 Lescoat A, Murphy SL, Roofeh D. Considerations for a combined index for limited cutaneous systemic sclerosis to support drug development and improve outcomes. J Scleroderma Relat Disord 2020;15.

16 Boers M, Kirwan JR, Gossec L, et al. How to choose core outcome measurement sets for clinical trials: OMERACT 11 approves filter 2.0 J Rheumatol 2014;41:1025-30.

17 Arksey H, O’Malley L. Scoping studies: towards a methodological framework. Int J Soc Res Methodol 2005;8:19-32.

18 Levac D, Colquhoun H, O'Brien KK. Scoping studies: advancing the methodology. Implement Sci 2010;5:69.

19 Munn Z, Peters MDJ, Stern C, et al. Systematic review or scoping review? Guidance for authors when choosing between a systematic or scoping review approach. BMC Med Res Methodol 2018;18:143.

20 Boers M, Beaton DE, Shea BJ, et al. OMERACT Filter 2.1: elaboration of the conceptual framework for outcome measurement in health intervention studies. J Rheumatol 2019;46:1021-7.

21 Khanna D, Krishnan E, Dewitt EM, et al. The future of measuring patient-reported outcomes in rheumatology: Patient-Reported Outcomes Measurement Information System (PROMIS). Arthritis Care Res 2011;63:S486-90.

22 Lescoat A, Cavalin C, Ehrlich R, et al. The nosology of systemic sclerosis: how lessons from the past offer new challenges in reframing an idiopathic rheumatological disorder. Lancet Rheumatol 2019;1:e257-64.

23 Wigley FM, Wise RA, Seibold JR, et al. Intravenous iloprost infusion in patients with Raynaud phenomenon secondary to systemic sclerosis. A multicenter, placebo-controlled, double-blind study. Ann Intern Med 1994;120:199-206.

24 Clements P, Lachenbruch P, Siebold J, et al. Inter and intraobserver variability of total skin thickness score (modified Rodnan TSS) in systemic sclerosis. J Rheumatol 1995;22:1281-5.

25 Steen VD, Medsger TA. The value of the health assessment questionnaire and special patient-generated scales to demonstrate change in systemic sclerosis patients over time. Arthritis Rheum 1997;40:1984-91.

26 Korn JH, Mayes M, Matucci Cerinic M, et al. Digital ulcers in systemic sclerosis: prevention by treatment with bosentan, an oral endothelin receptor antagonist. Arthritis Rheum 2004;50:3985-93.

27 Gliddon AE, Doré CJ, Black CM, et al. Prevention of vascular damage in scleroderma and autoimmune Raynaud's phenomenon: a multicenter, randomized, double-blind, placebo-controlled trial of the angiotensin-converting enzyme inhibitor quinapril. Arthritis Rheum 2007;56:3837-46.

28 Clements PJ, Roth MD, Elashoff R, et al. Scleroderma lung study (SLS): differences in the presentation and course of patients with limited versus diffuse systemic sclerosis. Ann Rheum Dis 2007;66:1641-7.

29 Nihtyanova SI, Brough GM, Black CM, et al. Clinical burden of digital vasculopathy in limited and diffuse cutaneous systemic sclerosis. Ann Rheum Dis 2008;67:120-3.

30 Hachulla E, Hatron P-Y, Carpentier P, et al. Efficacy of sildenafil on ischaemic digital ulcer healing in systemic sclerosis: the placebocontrolled SEDUCE study. Ann Rheum Dis 2016;75:1009-15.

31 Cutolo M, Herrick AL, Distler O, et al. Nailfold videocapillaroscopic features and other clinical risk factors for digital ulcers in systemic sclerosis: a multicenter, prospective cohort study. Arthritis Rheumatol 2016;68:2527-39.

32 Denton CP, Hachulla Éric, Riemekasten G, et al. Efficacy and safety of selexipag in adults with raynaud's phenomenon secondary to systemic sclerosis: a randomized, placebo-controlled, phase II study. Arthritis Rheumatol 2017;69:2370-9.

33 Methley AM, Campbell S, Chew-Graham C, et al. PICO, PICOS and SPIDER: a comparison study of specificity and sensitivity in three search tools for qualitative systematic reviews. BMC Health Serv Res 2014;14:579.

34 Avouac J, Fransen J, Walker UA, et al. Preliminary criteria for the very early diagnosis of systemic sclerosis: results of a Delphi Consensus Study from EULAR Scleroderma Trials and Research Group. Ann Rheum Dis 2011;70:476-81.

35 Tricco AC, Lillie E, Zarin W, et al. PRISMA Extension for Scoping Reviews (PRISMA-ScR): checklist and explanation. Ann Intern Med 2018;169:467-73.

36 Shamseer L, Moher D, Clarke M, et al. Preferred reporting items for systematic review and meta-analysis protocols (PRISMA-P) 2015: elaboration and explanation. BMJ 2015;350:g7647.

37 Jadad AR, Moore RA, Carroll D, et al. Assessing the quality of reports of randomized clinical trials: is blinding necessary? Control Clin Trials 1996;17:1-12.

38 Sterne JAC, Savović J, Page MJ, et al. Rob 2: a revised tool for assessing risk of bias in randomised trials. BMJ 2019;366:14898. 
39 Stang A. Critical evaluation of the Newcastle-Ottawa scale for the assessment of the quality of nonrandomized studies in meta- analyses. Eur J Epidemiol 2010;25:603-5. 